$$
\begin{aligned}
& \text { تقيم أداء الوحدات التوليدية المركبة باستخدام تقتية حقن البخار }
\end{aligned}
$$

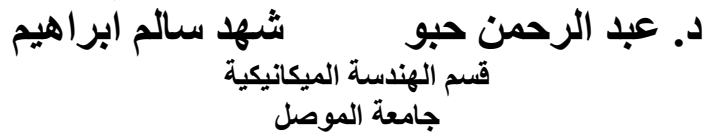

$$
\begin{aligned}
& \text { الخلاصة }
\end{aligned}
$$

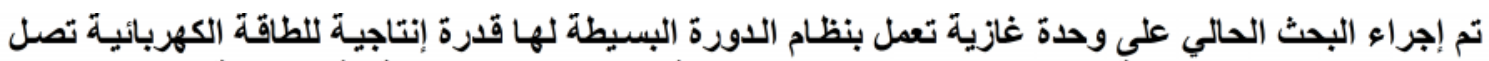

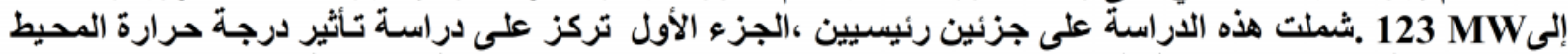

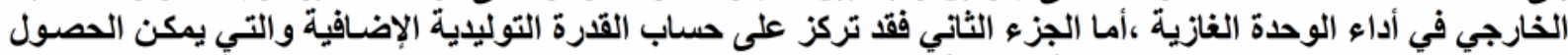

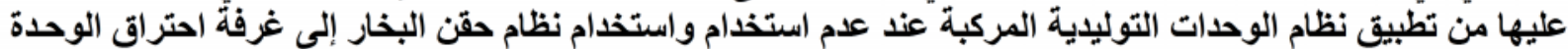

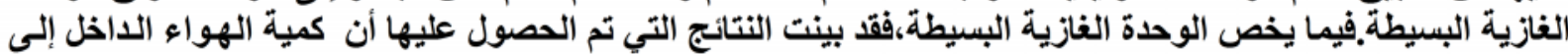

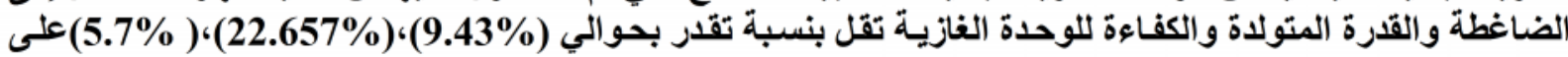

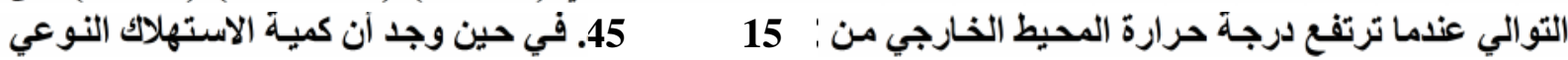
للوقود تزداد بنسبة قدرها (8\%)عندما ترتفع درجة حرارة المحيط الخارجي من التونة

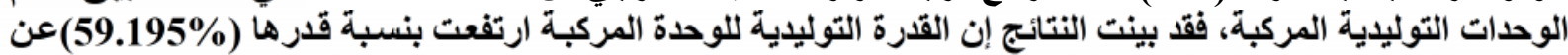

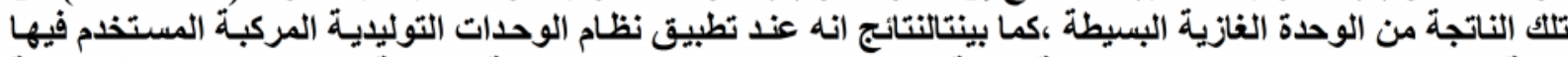

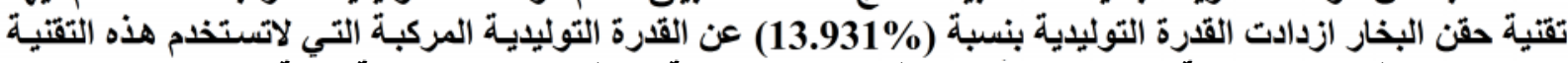

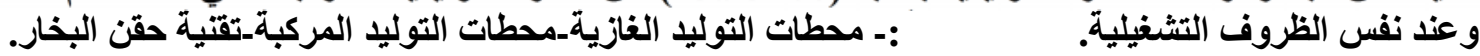

\title{
Evaluation of Performance of Combined Gas Units Using Steam Injection Technique
}

\author{
Dr. A. Haboo Sh. S. Ibrahim \\ Mechanical Engineering Department \\ University of Mosul
}

\section{Abstract}

The present work has been carried out on a gas turbine unit of a power output of 123MW operates on a simple cycle mode. The study involves two main parts. The first part has been focused on the effect of the ambient temperature rise on the performance of simpleg as turbine unit, while the second part was concentrated to evaluate the extra power output that can obtained ,"with and without steam injection technique", was applied to the combustion chamber of the simple gas turbine unit. Regarding the simple gas turbine, results show that the compressor mass flow rate of air power output, and thermal efficiency decreases, nearly by $(\mathbf{9 . 4 3 \%}),(22.627 \%)$ and $(5.7 \%)$ respectively, as the ambient temperature increases from $15^{\circ} \mathrm{C}$ to $45{ }^{\circ} \mathrm{C}$. While, the specific fuel consumption increases by nearly $(8 \%)$, as the ambient temperature rises from $15^{\circ} \mathrm{C}$ $45^{\circ} \mathrm{C}$. However, when the combined cycle mode is applied, results show that the generated power output for the combined unit, has been increased nearly by $(59.195 \%)$ over that one generated from simple cycle unit. Results also showed that when steam injection is used, in the combined power units, the power generated was increased by

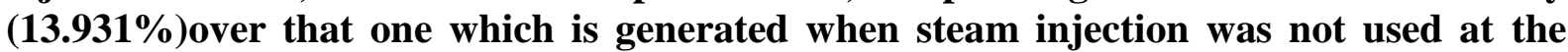
same operating condition. Key words:-Gas turbine, combined power plant, steam injection technique. 


\begin{tabular}{|c|c|c|c|}
\hline \multicolumn{2}{|c|}{ الرموز السفلية الدليلة } & \multirow[b]{2}{*}{ الضغط(bar) } & \multirow[b]{2}{*}{$\mathrm{P}$} \\
\hline الحالة عند النقطة. & 1,2, & & \\
\hline مضخة سائل التشغيل & Pum & نسبة الانضغاط & $r_{p c}$ \\
\hline الضاغطة & $\mathrm{C}$ & درجة الحرارة(C) & $\mathrm{T}$ \\
\hline التوربين الغازي. & GT & الحر ارة النوعية بثبوت الضغط(kJ/kg.K) & $\mathrm{C}_{\mathrm{P}}$ \\
\hline التوربين البخاري. & $\mathrm{ST}$ & معدل جريان الكتلة(kg/s) & $\mathrm{m}$ \\
\hline الصنافي الكلي للوحدة الغازية & net & نسبة الانخفاض في ضغط غرفة الاحتر اق & $\Delta \mathrm{P}_{\mathrm{cc}}$ \\
\hline غرفة الاحتر اق. & $\mathrm{cc}$ & القيمة الحر ارية الصغرى للوقود(kJ/kg) & LCV \\
\hline الهواء & $\mathrm{a}$ & درجة حرارة التضايق(Co') & $\Delta \mathrm{t}_{\mathrm{pp}}$ \\
\hline الوقود & $\mathrm{f}$ & درجة حرارة الاقتر اب(Co & $\Delta t_{a p p r}$ \\
\hline 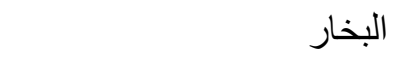 & $S$ & المساحة (m²) & A \\
\hline 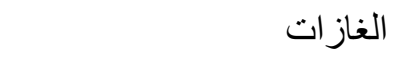 & $\mathrm{g}$ & السر عة (s / m ) & $\mathrm{V}$ \\
\hline 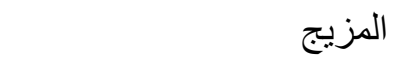 & $\mathrm{m}$ & المحتوى الحراري(kJ/kg ) & $\mathrm{H}$ \\
\hline الكلي & tot & نسبة الخسارة الحرارية في منظومة توليد & $\mathrm{h}_{\mathrm{l}}$ \\
\hline 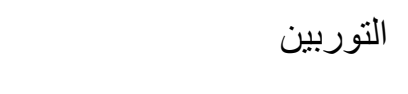 & $\mathrm{t}$ & الاختلاف بين درجة حرارة غاز ات العادم & TTD \\
\hline المستوى الوسطي & I & الطاقة الحر ارية(kJ) & Q \\
\hline مثالي & is & الشغل(kJ) & $\mathrm{W}$ \\
\hline 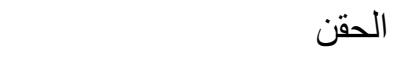 & inj & القدرة kW & $p$ \\
\hline & & الاستهلاك النوعي للوقودkg/kW.hr & SFC \\
\hline & & الوحدة الغازية|لمركبة بدون استخدام نظام الحقن & CCGTni \\
\hline & & الوحدة الغازية المركبة المطبق عليها نظام الحقن & CCGTwi \\
\hline \& & 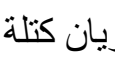 & النسبة بين معدل جريان كتلة البخار إلى معدل جر & $\mathrm{j}$ \\
\hline & & ين & موز - موز \\
\hline & & 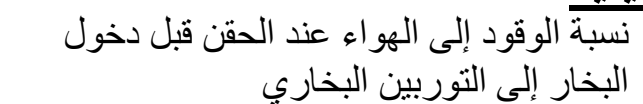 & $\bar{\lambda}$ \\
\hline & & الكفاءة & $\eta$ \\
\hline & & الكفاءة الميكانيكية & $\eta_{\mathrm{m}}$ \\
\hline & & الكثافة( & $\rho$ \\
\hline & & نسبة الحر ارة النوعية & $\gamma$ \\
\hline
\end{tabular}




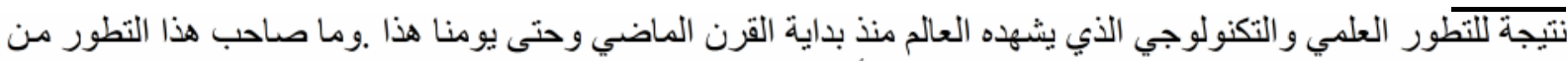

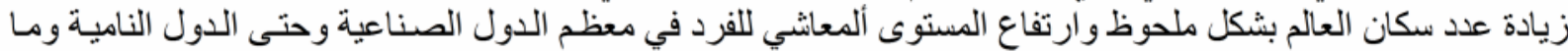

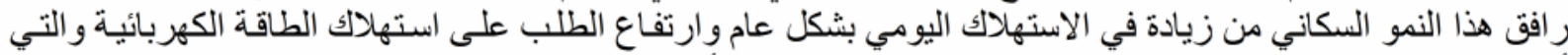

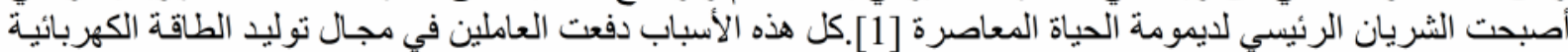

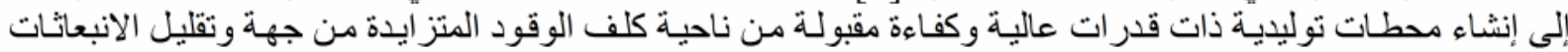

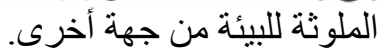

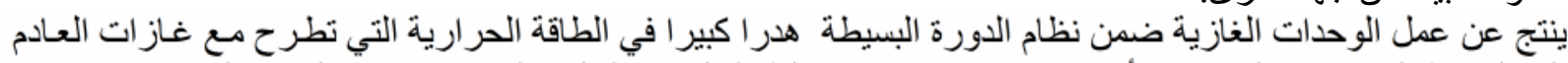

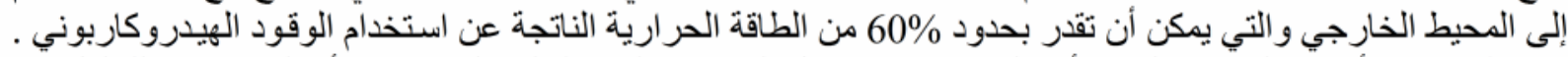

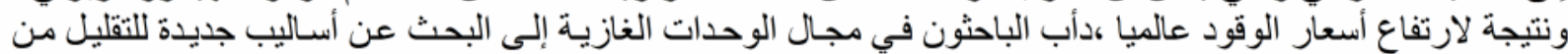

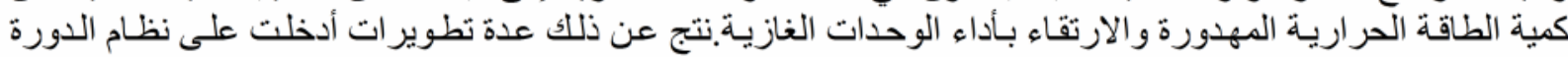

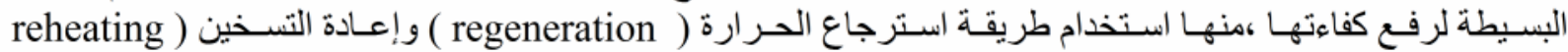

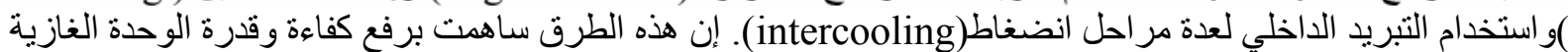

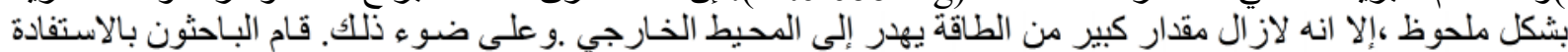

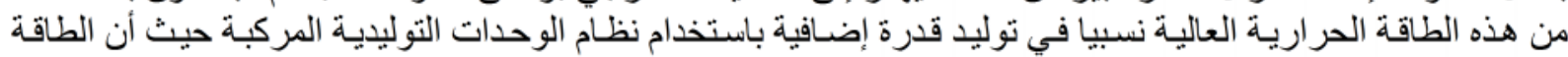

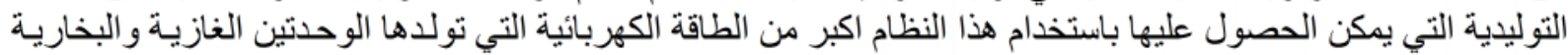

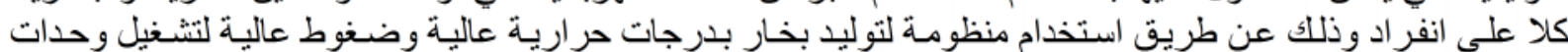

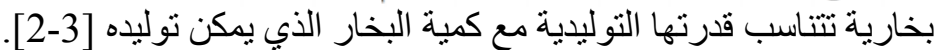

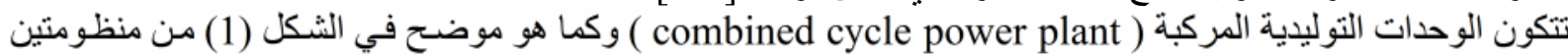

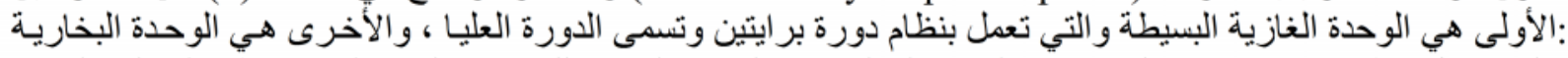

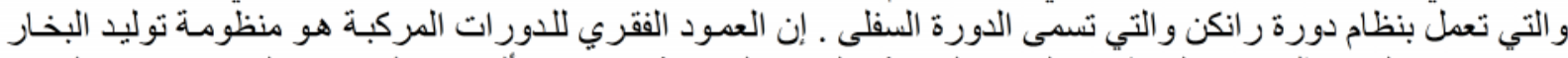

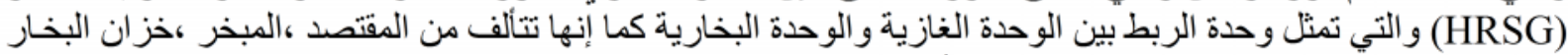

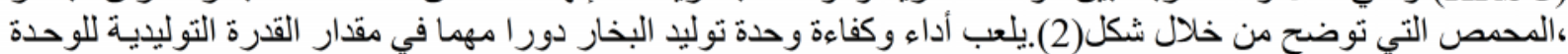

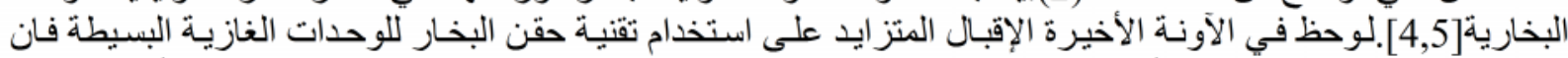

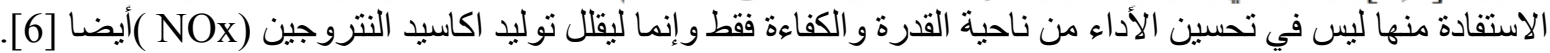

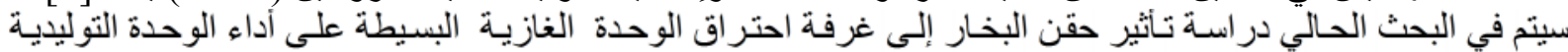

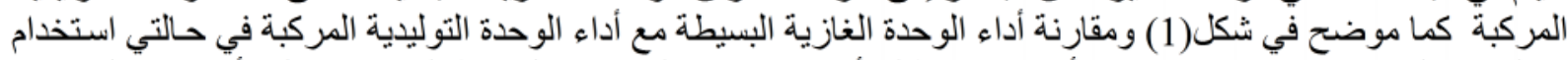

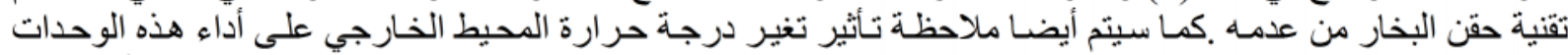

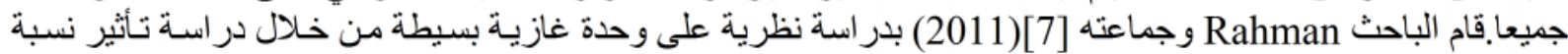

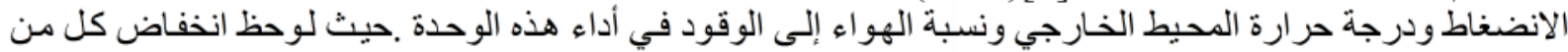

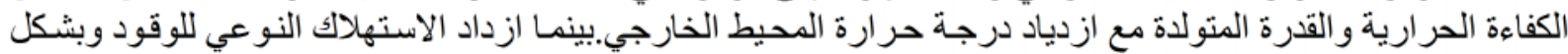

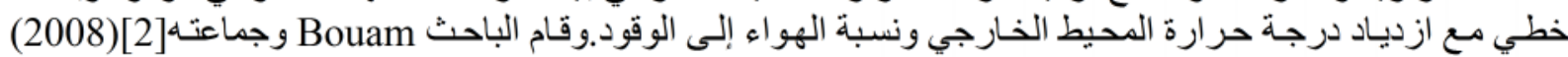

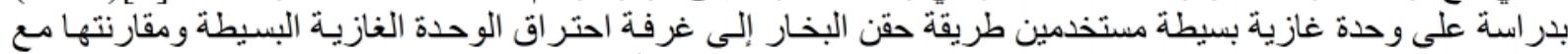

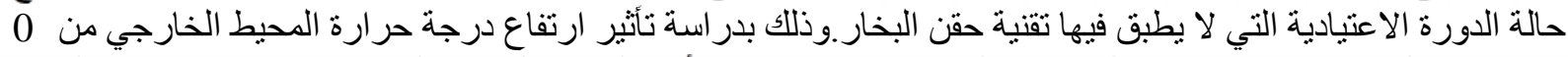
Cو وصو لا إلى

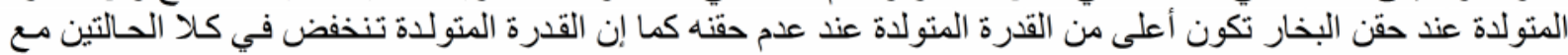

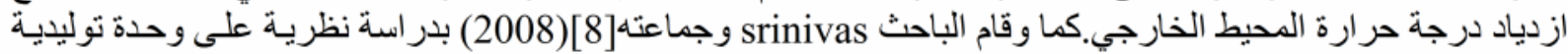

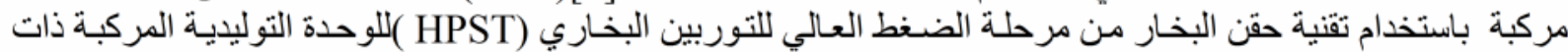

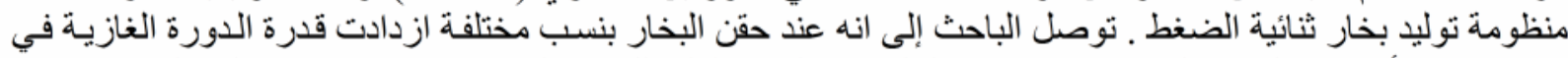

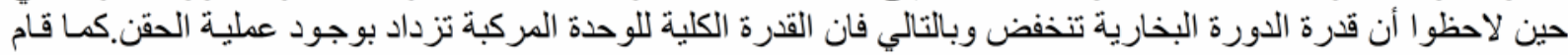

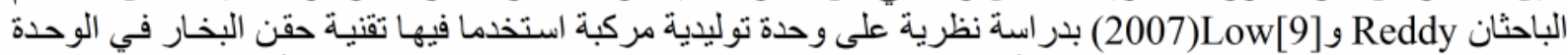

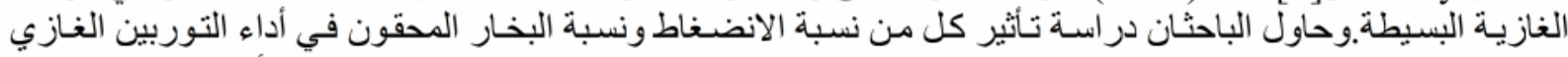

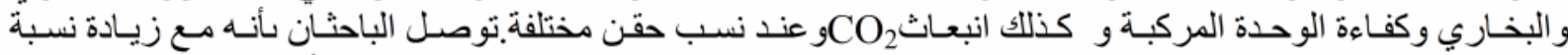

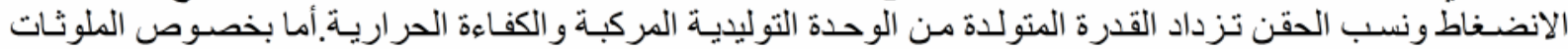

COيا 


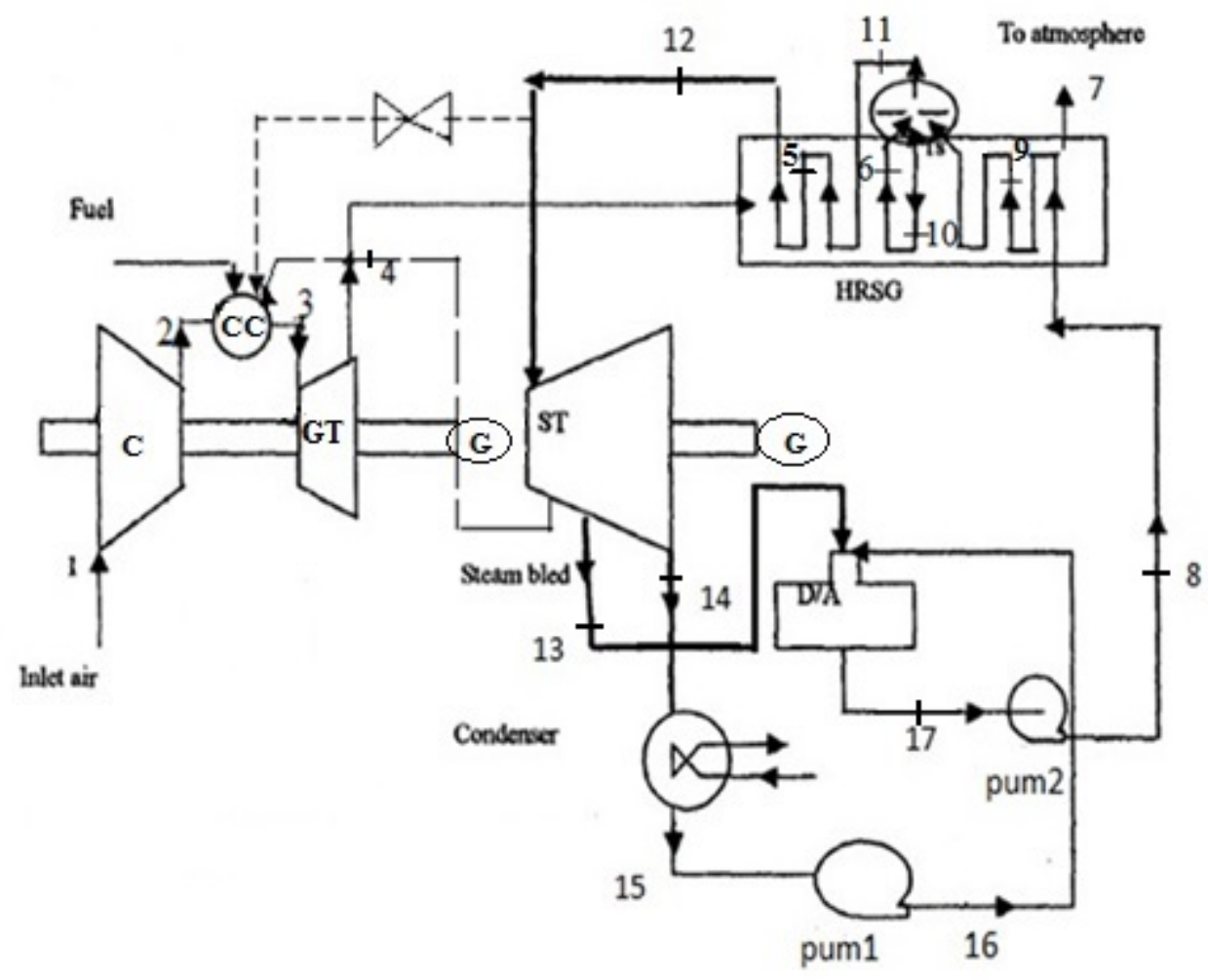

(1):الوحدة التوليدية المركبة في حالتي استخدام تقنية حقن البخار و عدم استخدامها
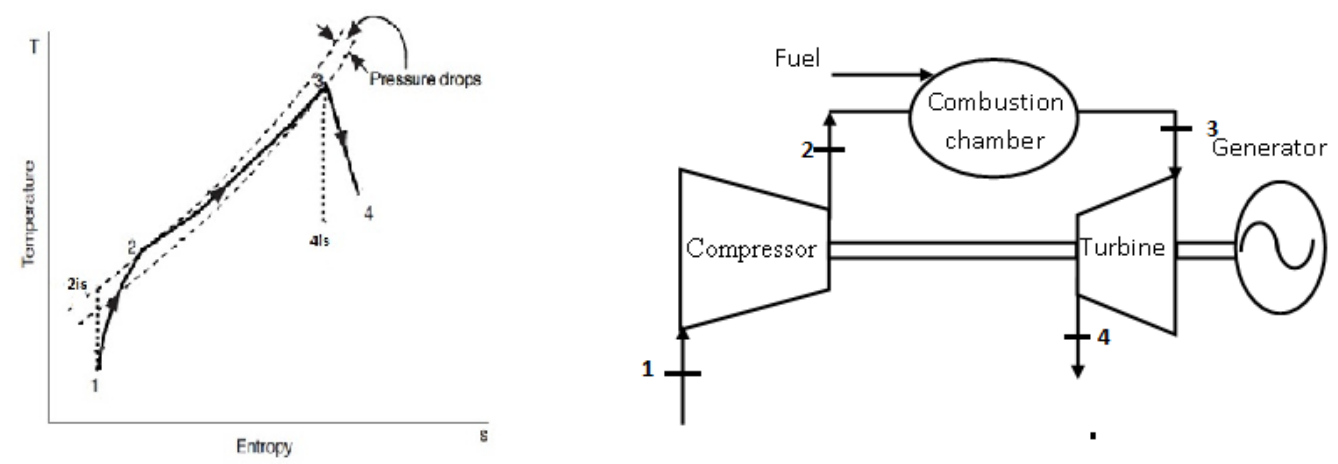

(2) :المكونات الرئيسية وعلاقة درجة الحرارة مع الانتروبي للوحدة الغازية البسيطة

تحليل

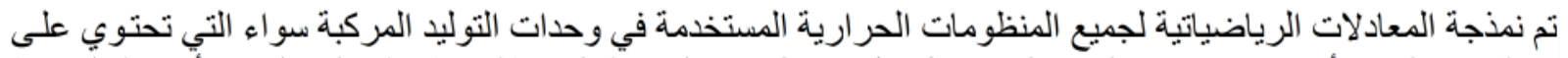

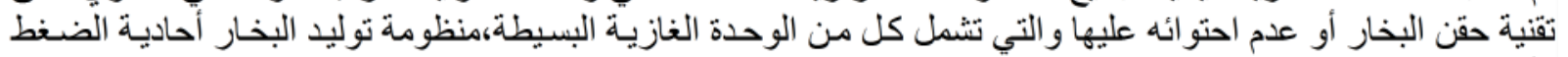

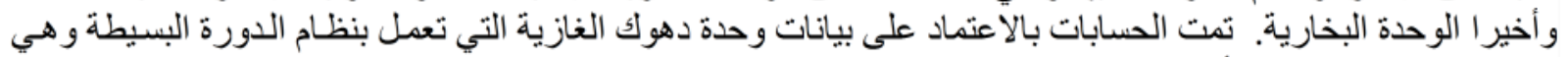

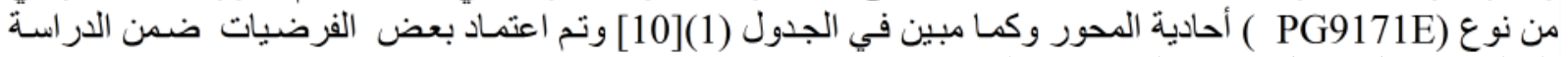

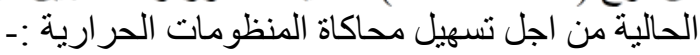

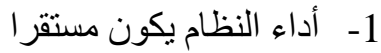




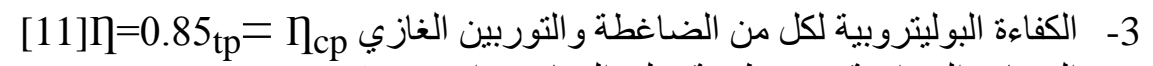

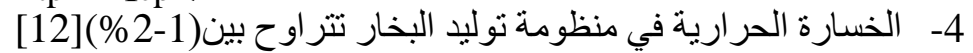

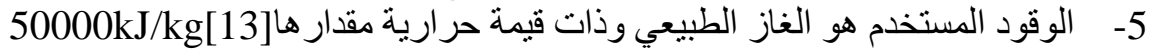

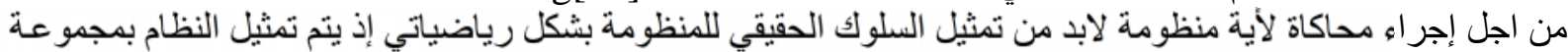

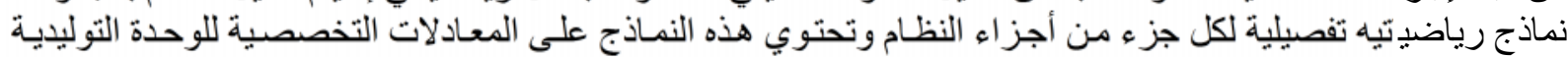
المحاكاة للمنظومات جميعا.

المركبة وتم الاستعانة ببرنامج حل المعادلات الهندسية (EES)

(1):مو اصفات الوحدة الغازية البسيطة

\begin{tabular}{|c|c|}
\hline PG9171E & نوع التوربين الغازي \\
\hline 100\%ميثان & \\
\hline $1.031 \mathrm{bar}$ & ضغط المحيط الخارجي \\
\hline 12.6 & \\
\hline $420.5(\mathrm{~kg} / \mathrm{s})$ & \\
\hline $8(\mathrm{~kg} / \mathrm{s})$ & \\
\hline $3000 \mathrm{rpm}$ & سر عة التوربين \\
\hline
\end{tabular}

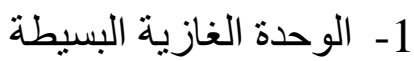

تلتكون الوحدات الغازية والتي تعمل بنظام الدورة البسيطة من ثلاثة أجزاء رئيسية :الضـاغطة ،غرفة الاحتر اق و التوربين

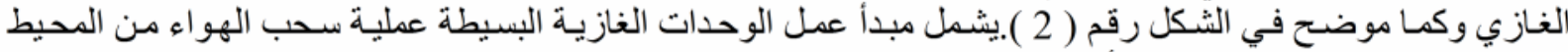

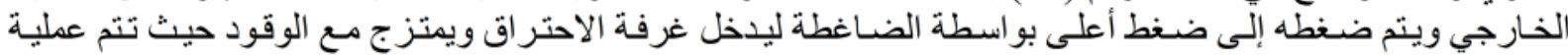

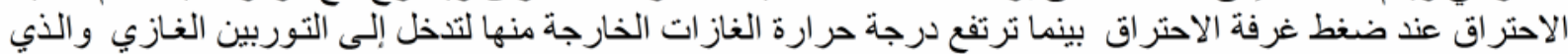

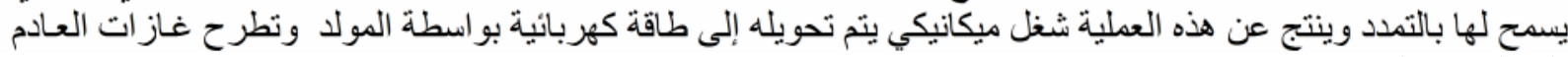

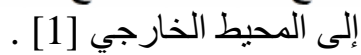

$\mathrm{P}_{2}=\mathrm{r}_{\mathrm{pc}} * \mathrm{P}_{1}$ ينم حساب ضغط الهو اء بعد خروجه من الضاغطة من المعادلة النالية : $\mathrm{T}_{2 \mathrm{is}}=\mathrm{T}_{1}\left(\mathrm{r}_{\mathrm{pc}}\right)^{\gamma_{\mathrm{a}}-1 / \gamma_{\mathrm{a}}}$

(2) $\mathrm{T}_{2}=\mathrm{T}_{1}+\frac{\left(\mathrm{T}_{2 \mathrm{is}}-\mathrm{T}_{1}\right)}{\mathrm{n} c}$

أما درجة الحر ارة الحققية الخارجة من الضاغطة فنحصل عليها من خلال استخدام المعادلة التالية: وان درجة الحر ارة المثالية الخارجة من الضاغطة يمكن حسابها من المعادلة أدناه: $\eta_{\mathrm{c}}=\frac{r_{p c}\left(\frac{\gamma a-1}{\gamma a}\right)-1}{r_{p c}\left(\frac{\gamma a-1}{\gamma a+\eta(p)}\right)-1}$ (isentropic efficiency)

$\eta_{\mathrm{t}}=\frac{1-\left(\frac{P_{4}}{P_{3}}\right) \frac{\eta_{\mathrm{tp}}\left(\gamma_{\mathrm{g}}-1\right)}{\gamma \mathrm{g}}}{1-\left(\frac{P_{4}}{P_{3}}\right) \frac{\left(\gamma_{\mathrm{g}}-1\right)}{\gamma \mathrm{g}}}$

$\dot{m}_{a}=\rho_{a} * \mathrm{~A} * \mathrm{~V}$

وكنلك لحساب معدل جريان كتلة الهو اء فقد تم استخدام المعادلة التالية:

$$
\mathrm{W}_{\mathrm{c}}=\mathrm{m}_{\mathrm{a}} \mathrm{C}_{\mathrm{Pa}}\left(\mathrm{T}_{2}-\mathrm{T}_{1}\right)
$$

بمكن حساب شغل الضاغطة من المعادلة النالية:

إن ضغط الغاز ات الخارجة من غرفة الاحتراق نحصل عليه من خلال المعادلة التالية[1]: 
$\mathrm{P}_{3}=\left(1-\Delta \mathrm{P}_{\mathrm{cc}}\right) * \mathrm{P}_{2}$

بينما يتم حساب درجة حرارة غاز ات العادم المثالية الخارجة من التوربين الغازي من خلال المعادلة التالية: $\mathrm{T}_{4 \text { is }}=\mathrm{T}_{3}\left[\frac{\mathrm{P}_{4}}{\mathrm{P}_{3}}\right]^{\frac{\gamma \mathrm{g}-1}{\gamma \mathrm{g}}}$

دلة التالية:

ولحساب درجة حرارة غاز ات العادم الحقيقية الخارجة من التوربين الغاز يتم

$\mathrm{T}_{4}=\mathrm{T}_{3}-\eta_{\mathrm{t}}\left(\mathrm{T}_{3}-\mathrm{T}_{4 \mathrm{is}}\right)$

2- الوحدات التوليدية المركبة المطبق عليها

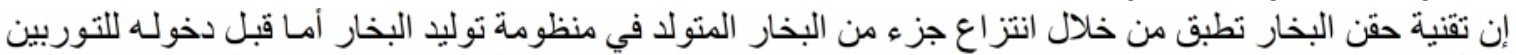

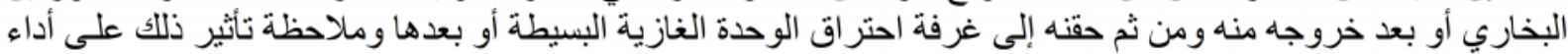

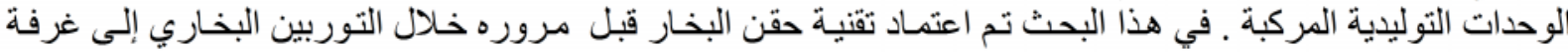
احتر اق الوحدة الغازية البسيطة مبانشرة.

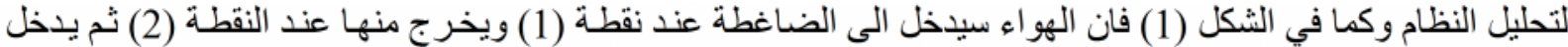

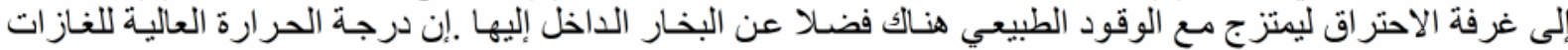

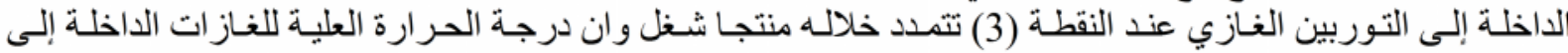

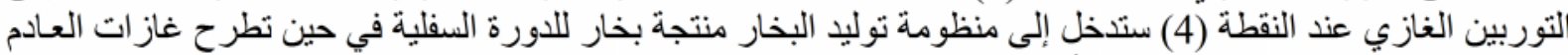

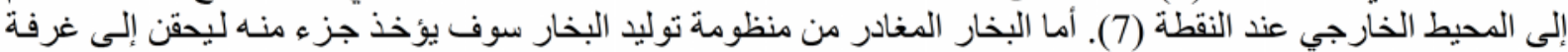

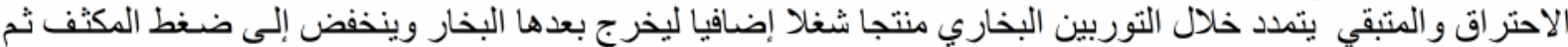

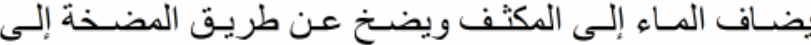

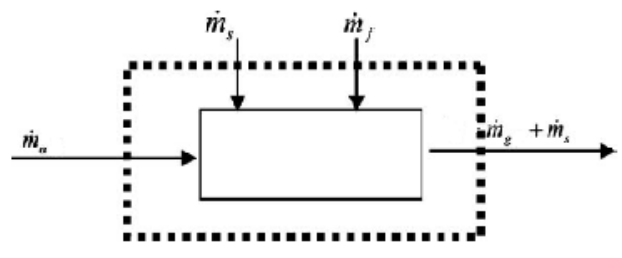

(3):غرفة احتر اق الوحدة الغازية البسيطة

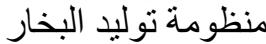
إن الفرق الرئيسي بين محاكاة الوحدات التوليدية المركبة التي

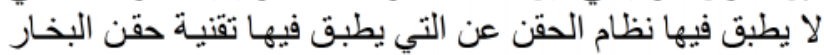

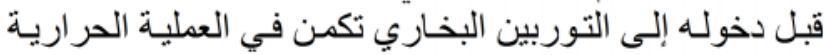

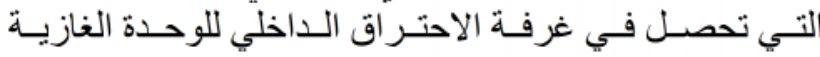

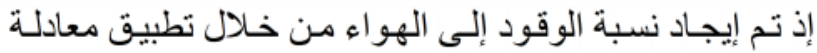

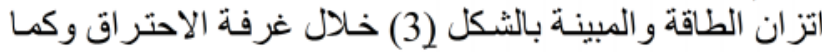
[2] مبين في المعادلة التالية

$\bar{\lambda}=\frac{\left(\mathrm{h}_{3 \mathrm{~g}}\right)-\left(\mathrm{h}_{2}\right)+\mathrm{j} *\left(\mathrm{~h}_{3 \mathrm{~s}}-\mathrm{h}_{\mathrm{inj}}\right)}{\mathrm{n}_{\mathrm{cc}} * \mathrm{LCV}-\left(\mathrm{h}_{3 \mathrm{~g}}\right)}$

$\mathrm{m}_{\overline{\mathrm{f}}}=\bar{\lambda} *$

وبالنسبة لكتلة الوقود الداخل إلى غرفة الاحتر اق فقد تم حسابه من المعادلة التالية: أما الكتلة الكلية المارة خلال غرفة الاحتر اق فيمكن إيجادها من المعادلة أدناه.

$\mathrm{m}_{\overline{\mathrm{tot}}}=\mathrm{m}_{\mathrm{a}}+\mathrm{m}_{\overline{\mathrm{f}}}+\mathrm{m}_{\mathrm{s}}$

$\mathrm{m}_{\mathrm{s}}=\mathrm{j} * \mathrm{~m}_{\mathrm{a}}$ و عليه فإن كمية البخار المحقون يمكن إيجادها من خلال المعادلة التالية:

في حين تم حساب المحتوى الحر اري للغاز ات المغادرة غرفة الاحتراق و الداخلة الى التوربين الغازي من خلال العلاقة

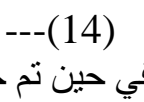

$\mathrm{h}_{3 \mathrm{~g}}=\mathrm{C}_{\mathrm{Pg}} * \mathrm{~T}_{3}$

$\mathrm{h}_{3 \mathrm{~s}}=\mathrm{h}\left(\mathrm{T}_{3}, \mathrm{P}_{3}\right)$ و وأما المحتوى الحراري للبخار المغادر غرفة الاحتراق فيتم إيجاده من العلاقة الآتية:

$\mathrm{h}_{\mathrm{inj}}=\mathrm{h}_{12}$ وفيما يخص المحتوى الحراري للبخار المحقون فيحسب من خلال العلاقة الآتية:

$\mathrm{W}_{\overline{\mathrm{net}}}=\mathrm{m}_{\overline{\mathrm{tot}}} \mathrm{C}_{\mathrm{Pm}}\left(\mathrm{T}_{3}-\mathrm{T}_{4}\right)-\mathrm{W}_{\mathrm{C}}$

بينما تم حساب شغل التوربين الغازي من خلال المعادلة الآتية. أما كمية الحر ارة المضافة نتيجة عملية احتر اق الوقود الهيدروكاربوني في غرفة الاحتر اق فقد تم حسابها كما يلي: 
$\mathrm{Q}_{\overline{\mathrm{f}}}=\mathrm{m}_{\overline{\mathrm{tot}}} \cdot \mathrm{C}_{\mathrm{Pm}} \cdot\left(\mathrm{T}_{3}-\mathrm{T}_{2}\right) \cdot \eta_{\mathrm{CC}}$

$\mathrm{C}_{\mathrm{Pm}}=\mathrm{C}_{\mathrm{Pg}}+j * \mathrm{C}_{\mathrm{Ps}}$

علما أن الحرارة النوعية للمزيج تم حسابها باستخدام المعادلة التالية:[14]

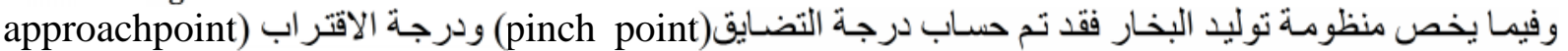
) واللتان يمكن تعريفهما كما موضح في الثكل (4)كما يتم الحصول عليها من خلال استخدام المعادلتين التاليتين:

$\Delta \mathrm{t}_{\mathrm{pp}}=\mathrm{T}_{6}-\mathrm{T}_{10}$

$\Delta \mathrm{t}_{\mathrm{appr}}=\mathrm{T}_{10}-\mathrm{T}_{9}$

وان درجة حر ارة البخار المحص تحسب من العلاقة أدناه بعد اخذ بنظر الاعتبار الفرق ثابت بين درجة حر ارة البخار

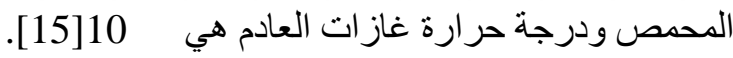

$\mathrm{T}_{12}=\mathrm{T}_{4}-\mathrm{TTD}$

في حين تم حسـاب كميـة البخـار المتولدة من منظومـة توليد البخـار من خـلال تطبيق معادلة اتز ان الطاقة بين منطقتي

$\mathrm{m}_{\overline{\mathrm{ST}}}=\frac{\mathrm{m}_{\mathrm{tot}} \mathrm{C}_{\mathrm{Pm}}\left(\mathrm{T}_{4}-\mathrm{T}_{6}\right)\left(1-\mathrm{h}_{1}\right)}{\mathrm{h}_{12}-\mathrm{h}_{9}}$

وان درجة حرارة الغاز ات المطروحة إلى المحيط الخارجي ستتأثر بتقنية الحقن وستحسب من المعادلة الآتية:

$\mathrm{T}_{\overline{7}}=\mathrm{T}_{4}-\left[\frac{\mathrm{m}_{\overline{\mathrm{ST}}} *\left(\mathrm{~h}_{9}-\mathrm{h}_{8}\right)}{\mathrm{m}_{\overline{\mathrm{tot}}}^{-} \mathrm{C}_{\mathrm{Pm}}\left(1-\mathrm{h}_{\mathrm{l}}\right)}\right]$

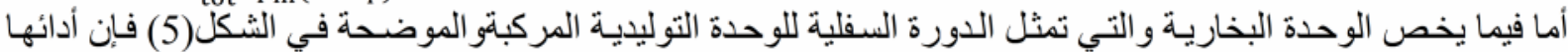

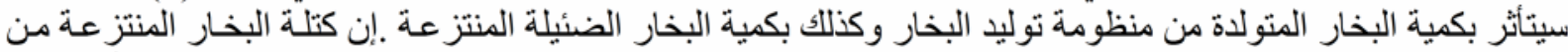

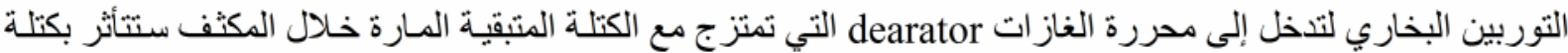
من منظومة توليد البخار الجديدة عند الحقن و تحسب من خلال استخدام المعادلة التالية.

$\mathrm{m}_{\overline{\mathrm{l}}}=\frac{\left(\mathrm{h}_{17}-\mathrm{h}_{15}\right) * \mathrm{~m}_{\overline{\mathrm{ST}}}}{\mathrm{h}_{13}-\mathrm{h}_{16}}$

وباستخدام المعادلة التالية سيتم حساب شغل التوربين البخاري:

$\mathrm{W}_{\overline{\mathrm{ST}}}=\left(\mathrm{m}_{\overline{\mathrm{ST}}}-\mathrm{m}_{\mathrm{s}}\right)\left(\mathrm{h}_{12}-\mathrm{h}_{13}\right)+\left(\mathrm{m}_{\overline{\mathrm{ST}}}-\mathrm{m}_{1}-\mathrm{m}_{\mathrm{s}}\right)\left(\mathrm{h}_{13}-\mathrm{h}_{14}\right)$

كما إن الثغل المبذول خلال المضختين يحسب من المعادلة التنالية:

$\mathrm{W}_{\overline{\text { pumtot }}}=\left(\mathrm{m}_{\overline{\mathrm{ST}}}-\mathrm{m}_{\mathrm{s}}\right)\left(\mathrm{h}_{8}-\mathrm{h}_{17}\right)+\left(\mathrm{m}_{\overline{\mathrm{ST}}}-\mathrm{m}_{\overline{\mathrm{i}}}-\mathrm{m}_{\mathrm{s}}\right)\left(\mathrm{h}_{15}-\mathrm{h}_{16}\right)$

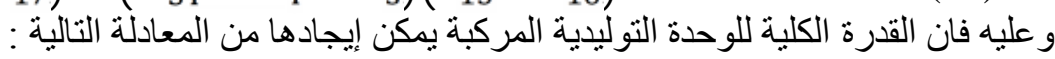

$\mathrm{p}_{\overline{\mathrm{CCGT}}}=\left(\mathrm{W}_{\overline{\mathrm{net}}} * \eta_{\mathrm{mGT}}\right)+\left(\left(\mathrm{W}_{\overline{\mathrm{ST}}} * \eta_{\mathrm{mST}}\right)-\left(\mathrm{W}_{\overline{\text { pumtot }}} * \eta_{\mathrm{mpum}}\right)\right)$

في حين يتم حساب الاستهلاك النوعي للوقود للوحدة التوليدية المركبة من المعادلة التالية:

$\mathrm{SFC}_{\overline{\mathrm{CCGT}}}=\frac{\dot{\mathrm{m}}_{\overline{\mathrm{f}}}{ }_{\overline{\mathrm{CCGT}}}}{\mathrm{p}_{\overline{\mathrm{C}}}}$

$\eta \overline{\mathrm{CCGT}}=\frac{\mathrm{p}_{\overline{\mathrm{CCGT}}}}{\mathrm{Q}_{\overline{\mathrm{f}}}}$

وتم إيجاد الكفاءة الحر ارية للوحدة التوليدية المركبة من المعادلة التالية:

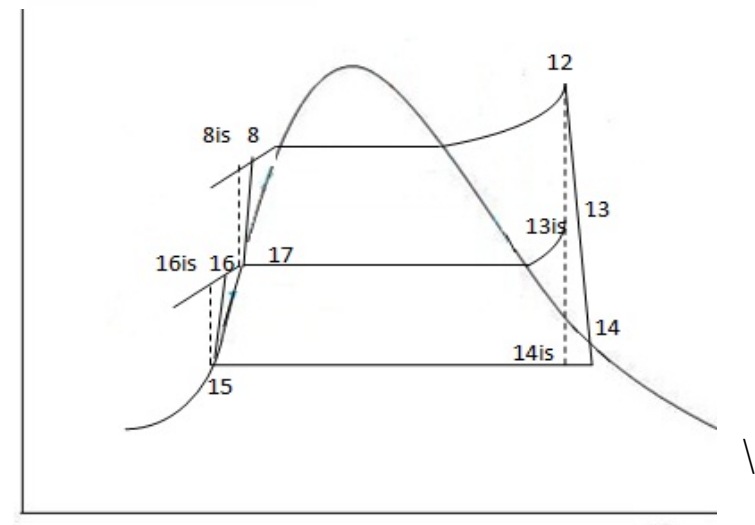

:(5)

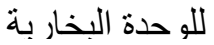

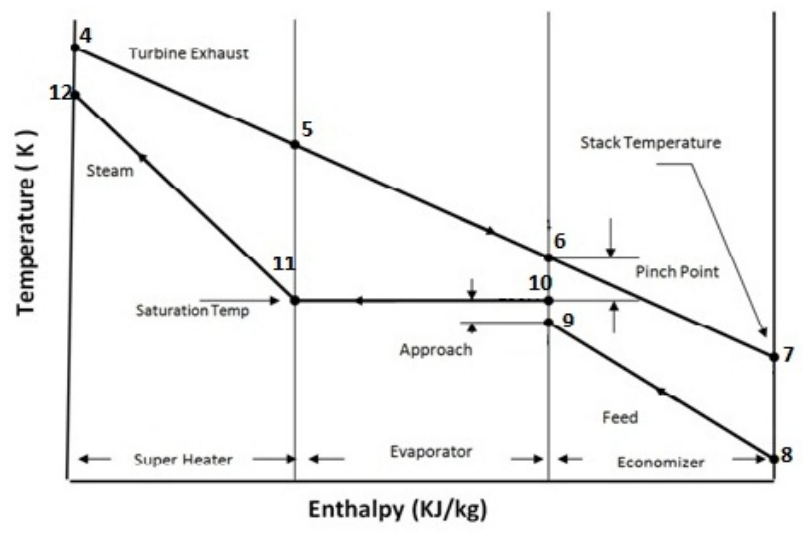

(4): توزيع درجات الحرارة خلال منظومة

توليد البخار أحادية الضغط 


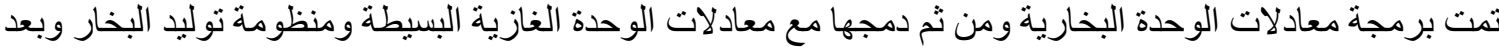

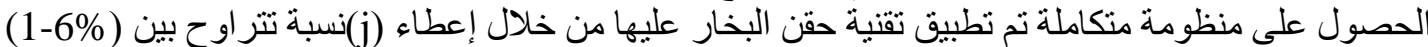

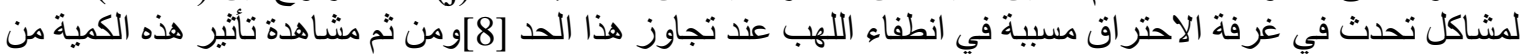

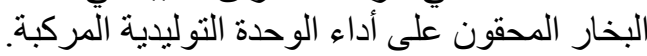

النتائج ومناقثتنها:

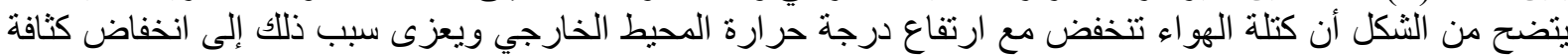

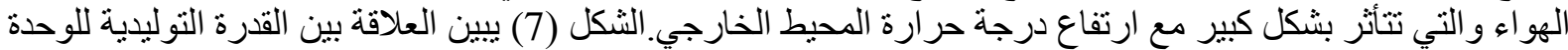

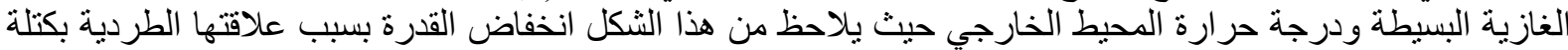

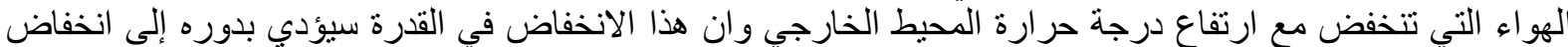

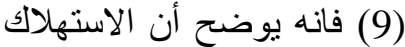

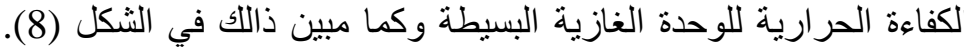

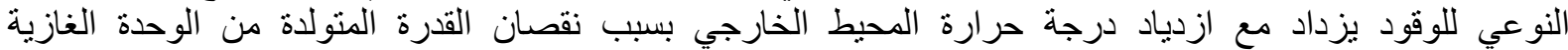

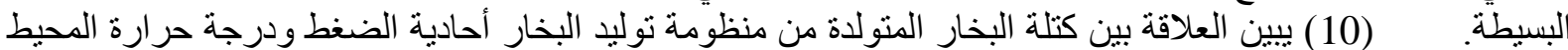

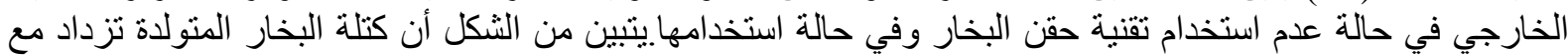

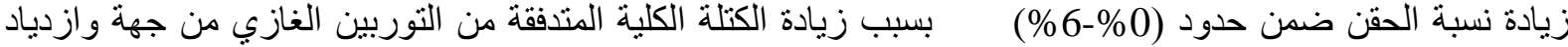

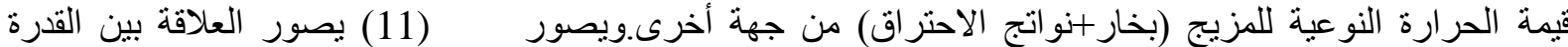

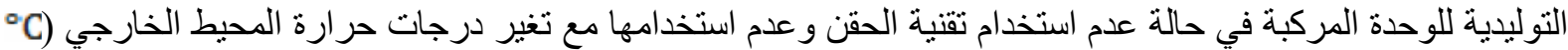

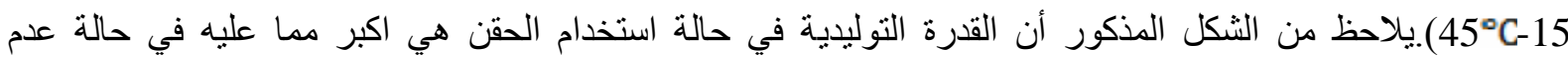

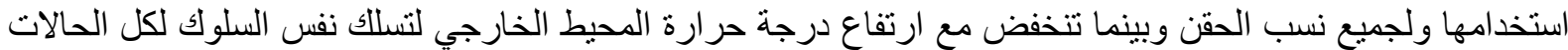

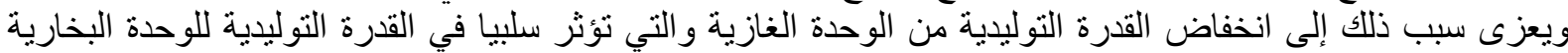

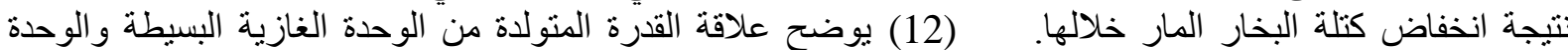

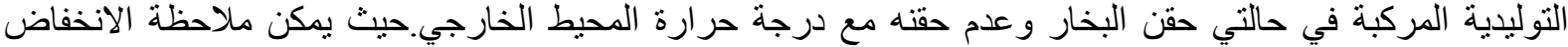

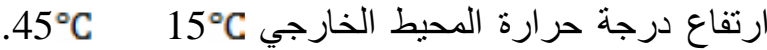

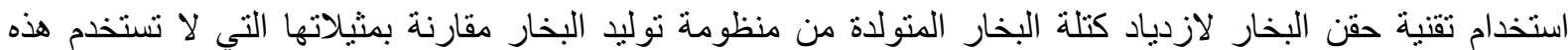

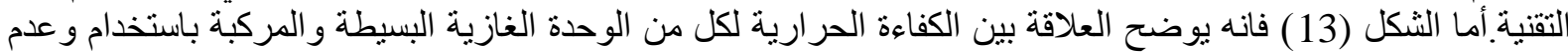

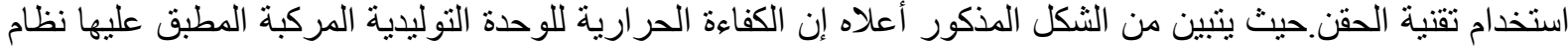

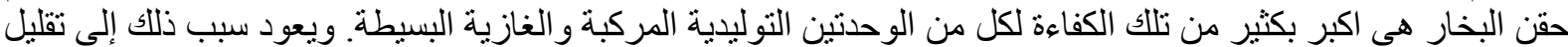

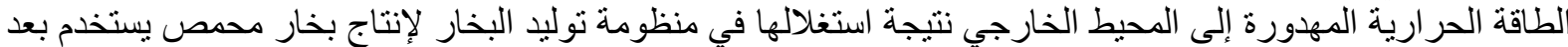

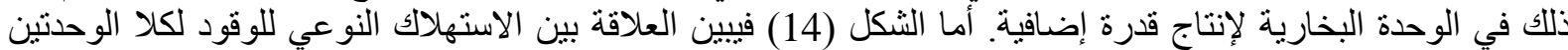

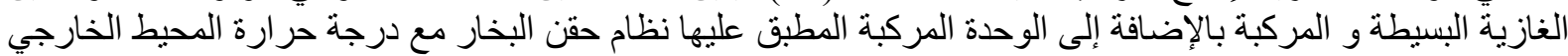

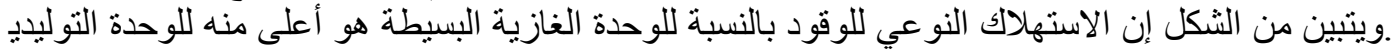

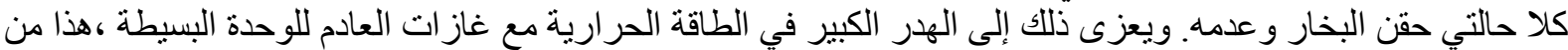

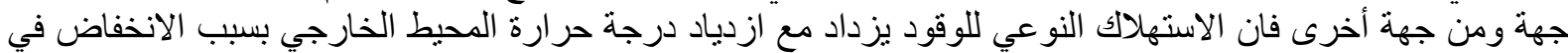

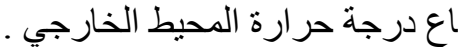




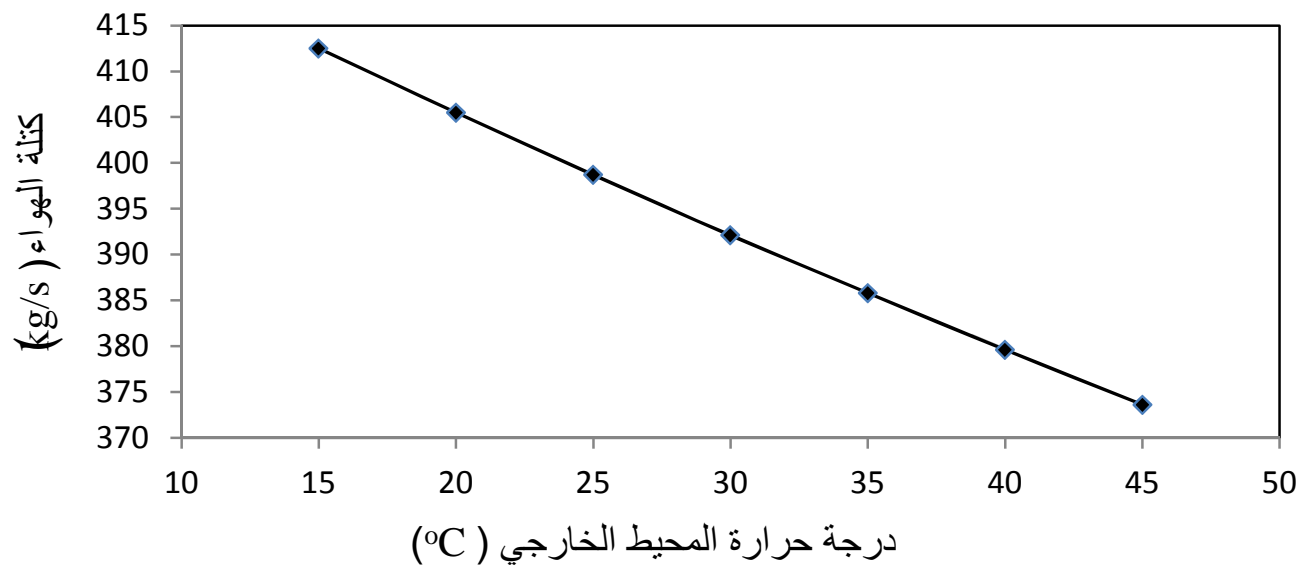

شكل(6):تاثير تغير درجة حر ارة المحيط الخارجي في كتلة الهو اء للوحدة الغازية البسيطة

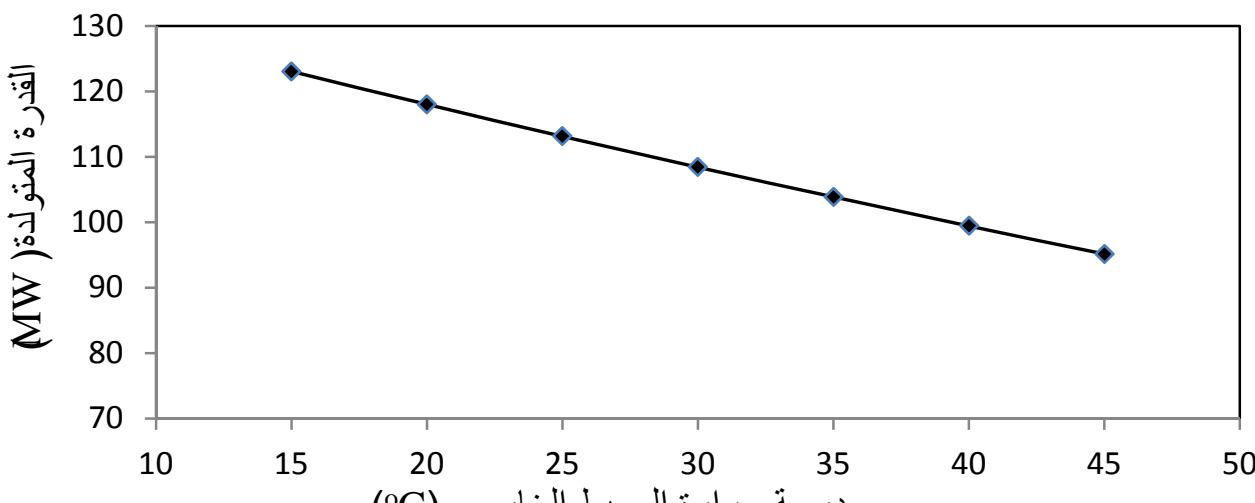

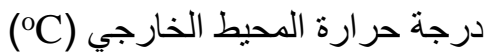

شكل(7):تاثير تغير درجة حرارة المحيط الخارجي في القدرة المتولدة للوحدة الغازية البسيطة

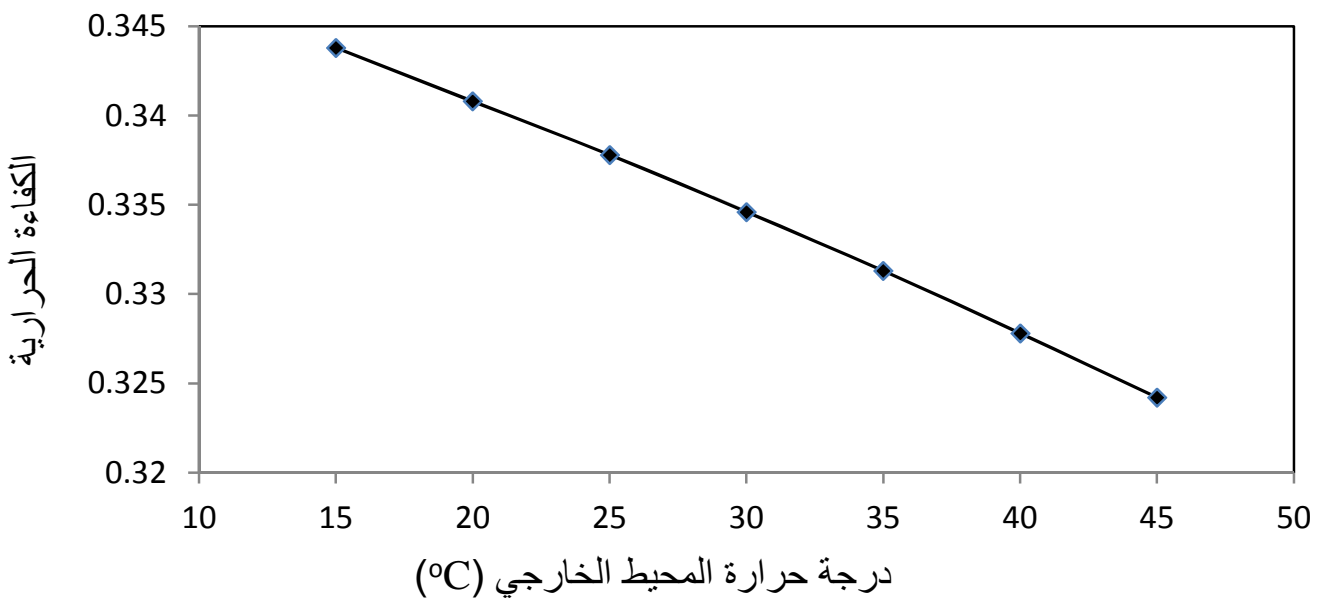

شكل(8):تاثير تغير درجة حرارة المحيط الخارجي في الكفاءة الحرارية 


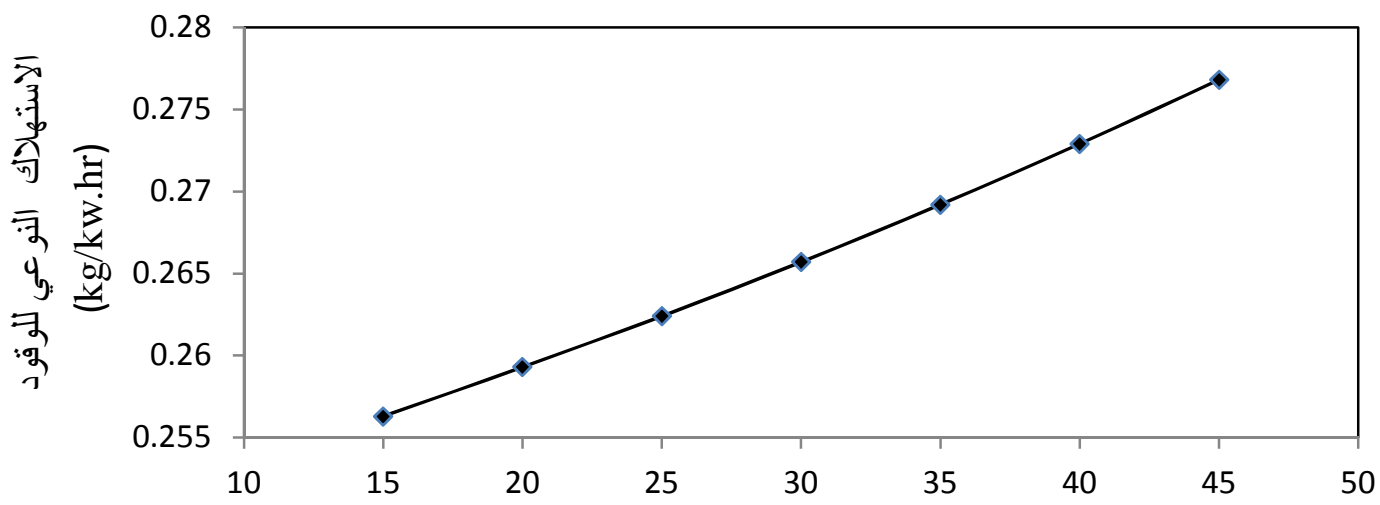

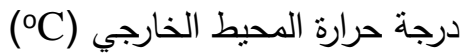

شكل(9):تاثير تغير درجة حرارة المحيط الخارجي في الاستهلاك النوعي للوقود للوحدة الغازية البسيطة

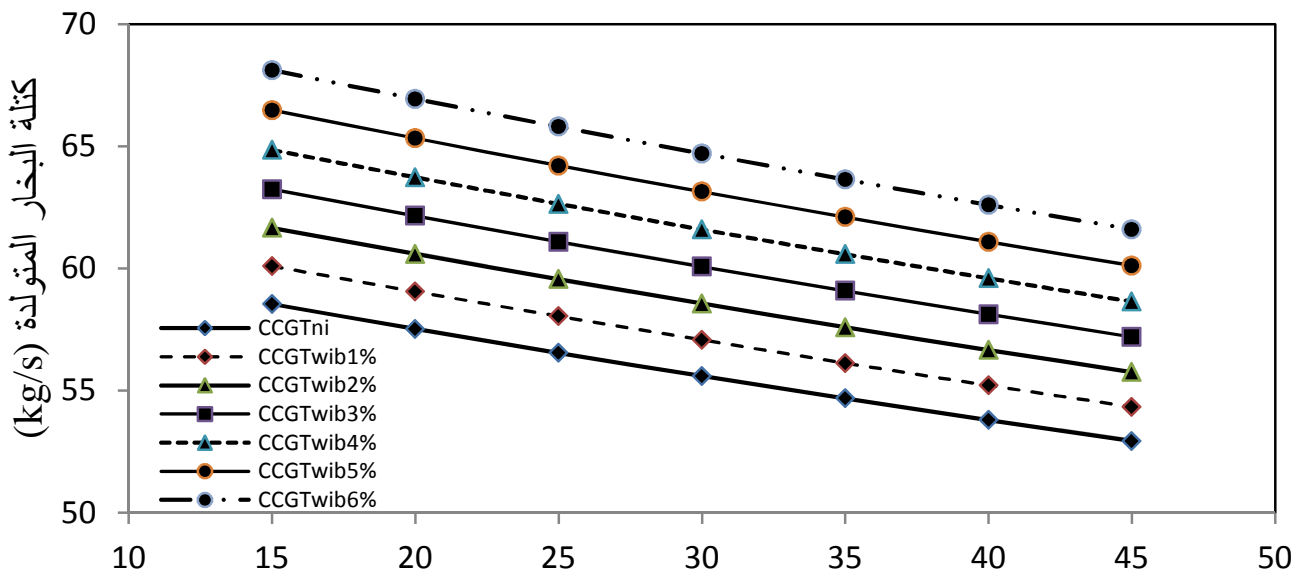

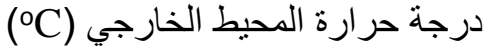

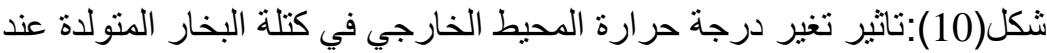
حقن البخار قبل دخوله الى التوربين البخاري

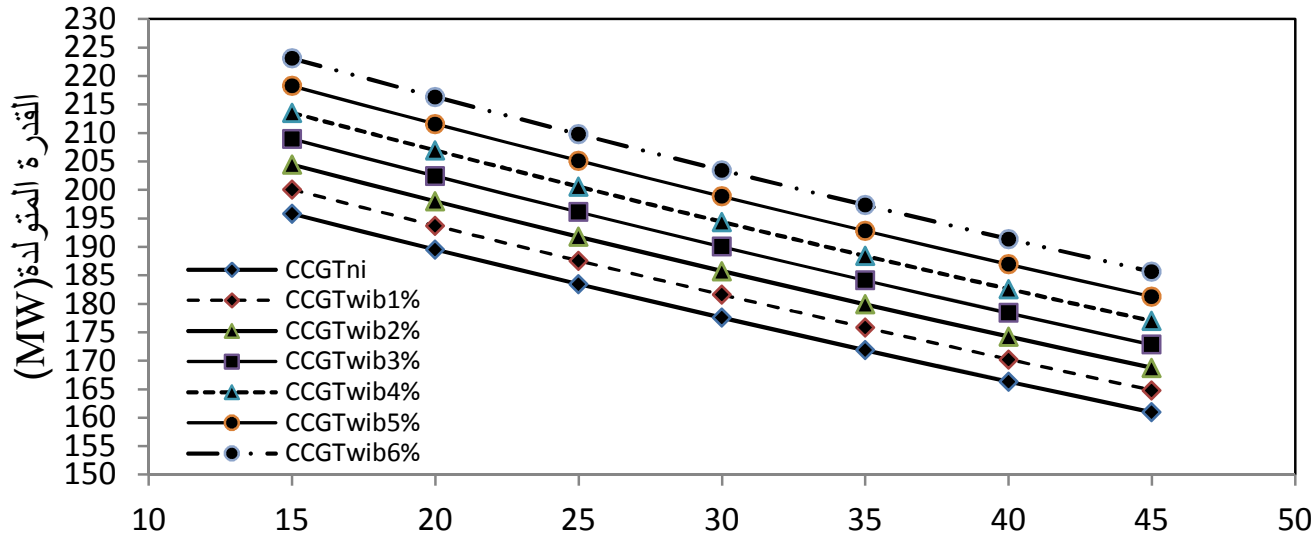

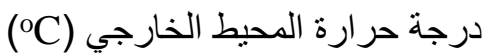

شكل(11):تاثير تغير درجة حرارة المحيط الخارجي في القدرة المتولدة عند حقن البخار قبل دخوله الى التوربين البخاري 
: تقييم أداء الوحدات التوليدية المركبة باستذام تقتية حقن البخار

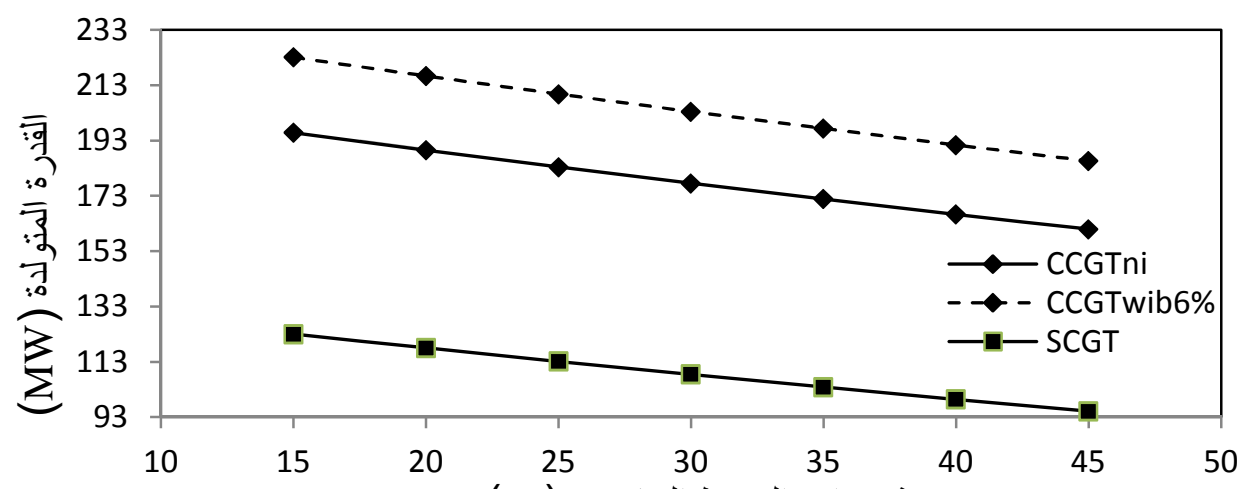

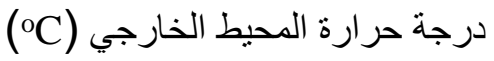

شكل (12):تاثير تغيردرجة حرارة المحيط الخارجي في القدرة المتولدة

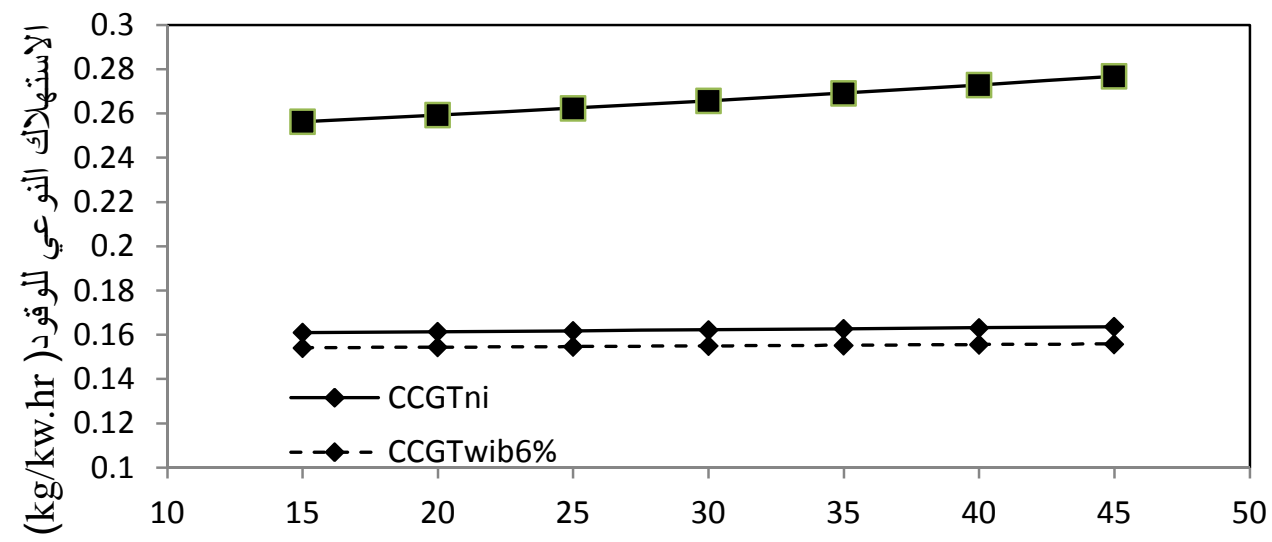

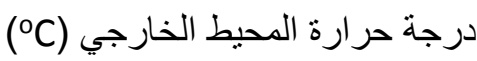

شكل (14):تاثير تغيردرجة حرارة المحيط الخارجي في الاستهلاك النوعي للوقود

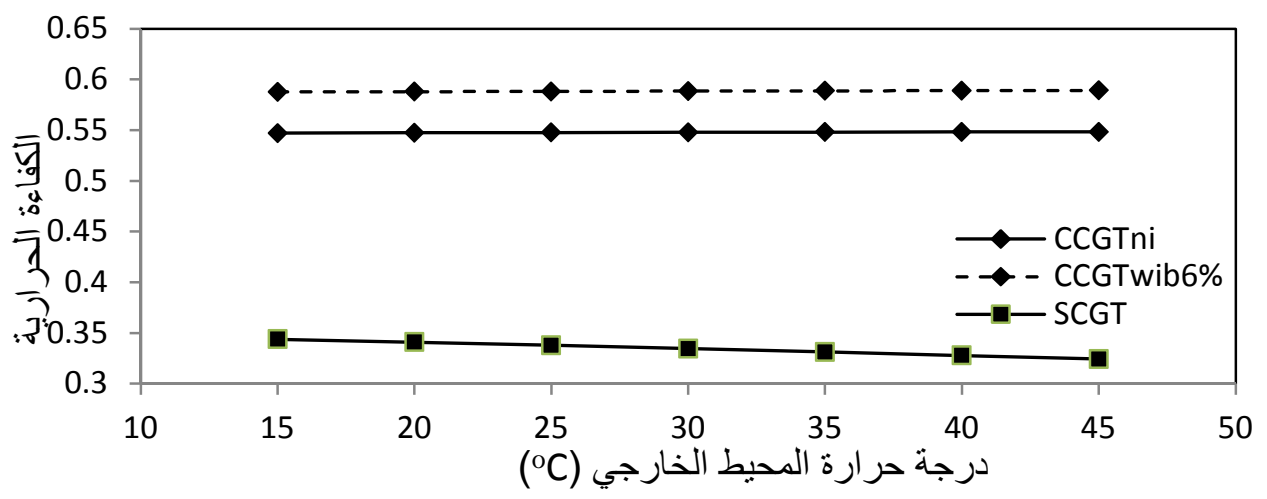

شكل (13) تاثير تغيردرجة حرارة المحيط الخارجي في الكفاءة الحرارية

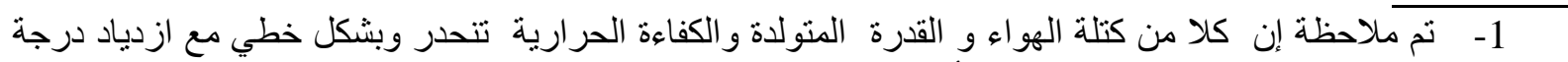

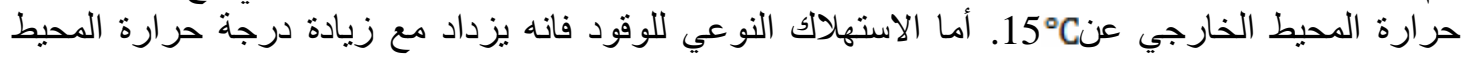

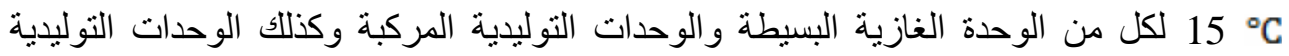

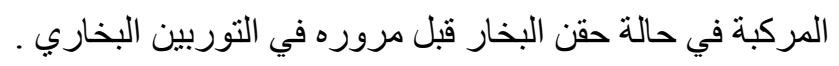


2- وجد إن تطبيق نظام الوحدات التوليديد الحرارية على الوحدة الغازية البسيطة يؤدي إلى زيادة واضحة

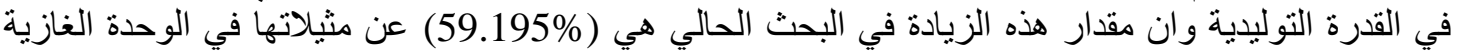

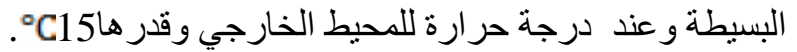

6\% 6لوحظ إن كلا من القدرة التوليدية و الكفاءة 3- في حالة استخدام تقنية حقن الحر ارية للوحدة المركبة تزداد بنسبة قدر ها (13.931\%) (7.421)على التو الي ن تللك الناتجة من الوحدة الغازية

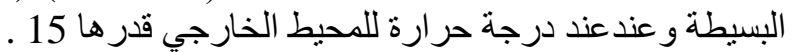

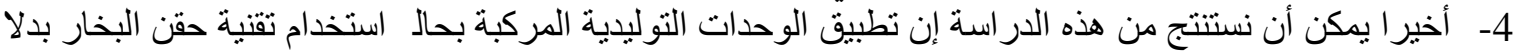

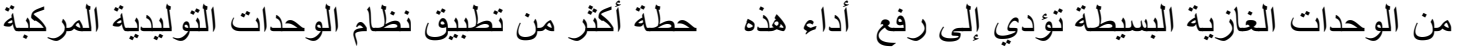
على الوحدات الغازية البسيطة.

[1] Kumer , N.R., Krishna ,K. R. and Rama ,A.V., “ Thermodynamic analysis of heat recovery steam generator in combined cycle power plants " Thermal science ,Vol. 11,No.4,pp. 143-156,2007.

[2] Bouam ., Aissani ,S. and Kadi ,R.," Combustion chamber steam injection for gas turbine performance improvement operations “Journal of engineering for gas turbine and power ,Vol.130,pp.041701-1,2008.

[3] Horlock,J.H.,"Advanced gas turbine cycle", Whittle laboratory Combringe, J.K.,1edition,2003.

[4] Carapellucci ,R.,"A unfired approach to assess performance of different techniques for recovering exhaust heat from gas turbine", Energy conversion and management, Vol.50,pp.1218-1226,2009.

[5] Korakianitis,T., Grantstrom ,J ., Wassingbo ,P., and Massardo ,F.A. ,"Parametric performance of combined-cogeneration power plant with various power and efficiency enhancements", Journal of engineering for gas turbine and power,Vol.127,pp.65-72,2005.

[6]Al Doori ,W.H.," Influence of steam injection on the performance of combined cycle power plant “,Journal of engineering and science, Vol.6,pp.390-396,2011.

[7] Rahman ,M.M., Ibrahim ,T,K., and Abdulla ,A,N.," Thermodynamic performance analysis of gas turbine power plant", International journal of the physical sciences, Vol.6,No.14,pp.3539-3550,2011.

[8] Srinivas ,T., Gupta ,S.V ., and ready ,V.B.," Sensitivity and analysis of SITG based combined cycle with dual pressure HRSG " International journal of thermal science , Vol.47,pp.1226-1234, 2008.

[9] Low ,B .Reddy, B, V., "Performance simulation of a combined cycle power generation system with steam injection in the gas turbine combustion chamber", proceedings of ES2007,pp.27-30,2007.

[10] General electric model PG9171E UP rate gas turbine Manual 2008.

[11] Srinivas ,T., "Study of a dearator location in triple pressure reheat combined powercycle“"Energy,vol.34,pp.1364-137,2009.

[12] Butcher, C.J, Reddey ,B.V.," Second low analysis of a waste heat recovery based power generation system", International Journal of heat and mass transfer vol.50,pp.23552363,(2007). [13] Colin R., Ferguson., Allain T. and Kirkpatrick," Internal combustion engine", John Wiley a sons,Inc.,2001.

[14] Kenneth C. Weston., "Energy conversion" chapter 9,pp.333-394,Advance system ,2000.

[15] Cogeneration and combined cycle principles http://www.hrsgdesign.com/design .htm.

$$
\text { تم اجراء البحث في كلية ألهندسة = جامعة ألموصل }
$$

